\title{
ANÁLISIS DE ESTABILIDAD DE TALUDES DE LA ZONA 'LOS BALUARTES' EN EL PARQUE ARQUEOLÓGICO DE SAQSAYHUAMÁN
}

\author{
Slope stability analysis of Los Baluartes area in the Archaeological Park of Saqsayhuaman
}

\author{
Diana Carmelí Ttito Ortiz ${ }^{1, a, \text {, }}$ \\ ${ }^{1}$ Universidad Andina del Cusco, Cusco, Perú. \\ a Bachiller en Ingeniería civil \\ ”dianattior@hotmail.com ; Av. Túpac Amaru V-1, Wánchaq, Cusco, Perú
}

\begin{abstract}
Resumen
El Parque Arqueológico de Saqsayhuamán es considerado 'Patrimonio Cultural de la Humanidad', por poseer características estructurales y arquitectónicas extraordinarias. Por lo cual, es de vital importancia su estudio desde un enfoque ingenieril para su conservación. En esta investigación se analizó la estabilidad de los taludes de la zona 'Los Baluartes', mediante el cálculo de factores de seguridad, obtención de parámetros de resistencia al corte del suelo y la determinación de otros factores influyentes en la estabilidad del talud (fuerzas sísmicas, factores hidrológicos, etc.). En tal sentido, este artículo presenta los resultados de los ensayos geotécnicos y geofísicos, además, el procesamiento de los datos obtenidos usando el programa Slide V.6, para el análisis seudoestático de los taludes. Cabe resaltar que esta investigación se desarrolló con el aporte de información y el trabajo realizado en el Parque Arqueológico de Saqsayhuamán, por la Universidad Nacional de Ingeniería, en los años 2016 y 2017

Palabras clave: Análisis de estabilidad, taludes, análisis seudoestático, Saqsayhuamán, superficie de falla, factor de seguridad
\end{abstract}

\section{Abstract}

Archaeological Park of Saqsayhuaman is considered like 'Cultural Heritage of Humanity' because of its extraordinary structural and architectural characteristics. Therefore, it is of vital importance to study it from an engineering perspective for its conservation. In this research, the slope stability analysis of Los Baluartes area in the Archaeological Park of Saqsaywaman was analyzed, by calculating safety factors, obtaining parameters of soil cutting resistance and determining other influential factors in the slope stability (seismic forces, hydrological factors, etc.). In addition, the paper presents results of the geotechnical and geophysical tests, and the processing of obtained data, using the Slide V.6 program for slope pseudostatic analysis. Besides, the study was developed with the contribution of information and work done in Archaeological Park of Saqsayhuaman, by the Universidad Nacional de Ingeniería, in 2016 and 2017.

Keywords: stability analysis, slopes, pseudostatic analysis, Saqsayhuaman, failure surface, safety factor

Citar como: Ttito, D. (2018). Análisis de estabilidad de taludes de la zona 'Los Baluartes' en el Parque Arqueológico de Saqsayhuamán. Rev Yachay, 7(1),322-333.

Recibido: 16-09-2018; Aceptado 07-12-2018

Rev. Yachay volumen (7) Número (1), enero-diciembre 2018 


\section{Introducción}

Cusco, además de ser calificada como la capital del imperio Inca, es cuna de grandes obras de ingeniería. Una de las más destacadas es la fortaleza de Sacsayhuamán (hoy 'parque arqueológico'), considerada Patrimonio Cultural de la Humanidad, por poseer características estructurales y arquitectónicas extraordinarias.

Uno de los sectores pertenecientes al Parque Arqueológico de Sacsayhuamán (PAS) es el sector 'Los Baluartes', compuesto por grandes muros zigzagueantes emplazados a lo largo de 500 metros en la meseta de Sacsayhuamán. Es de lamentar que, desde el año 2009, parte de estos muros (pertenecientes a la tercera terraza de dicha zona), colapsaron debido al derrumbe de algunos tramos del talud.

Ante esta situación la presente investigación analiza la estabilidad de los taludes de la zona 'Los Baluartes', mediante el cálculo de factores de seguridad, obtención de parámetros de resistencia al corte del suelo y la determinación de otros factores influyentes en la estabilidad del talud (fuerzas sísmicas, factores hidrológicos, etc.).

Finalmente, con el desarrollo de este estudio damos a conocer las zonas que presentan mayor riesgo de inestabilidad, así como los sectores que necesitan mayor atención e intervención, para poder evitar la pérdida de tan valioso legado inca. Del mismo modo, se proponen recomendaciones para futuras investigaciones y tenerlas de precedente para aquellos que deseen realizar mayores estudios en el Parque Arqueológico de Sacsayhuamán.

\section{El Parque Arqueológico de Sacsayhuamán}

Ubicado a dos kilómetros al noreste y por encima de la ciudad de Cusco, sobre la meseta del mismo nombre a una altura media de 3550 msnm; cuenta con un área de 3093.80 hectáreas y un perímetro de $26550 \mathrm{~m}$.

A su vez, la investigación analiza el sector de 'Los Baluartes', perteneciente al PAS y ubicado en la ladera septentrional de la meseta de Sacsayhuamán. Consta de muros monumentales de calizas labradas de grandes dimensiones, dispuestos en tres niveles con forma de zigzag.

\section{Aspectos históricos}

La tradición refiere que la construcción de Sacsayhuamán comenzó a fines del siglo XIV o a principios del siglo XV de nuestra era, fue emprendida por el décimo inca, Inca Yupanqui, y terminada durante el reinado de Huayna Cápac, de modo que se terminó de construir en el curso de unos 60 años. De acuerdo con algunos cronistas, los cimientos de Sacsayhuamán fueron colocados aún por el antecesor de Yupanqui, Pachacútec Inca (Middenfort, 1895).

Hoy en día, muchos investigadores descartan que Sacsayhuamán fuese construido para servir de fortaleza militar y proponen su interpretación como centro ceremonial y templo del sol. Es la idea que recoge Cieza de León, cuando afirma que los incas llamaban 'Casa del Sol' a lo que los españoles denominaron fortaleza (Mar \& Beltrán, 2014).

Garcilaso de la Vega, nombra a cuatro arquitectos: Apu Huallpa Rimachi, Inca Maricanchi, Acahuana Inca y Callacunchuy, quienes dirigieron sucesivamente la obra (M. Rivero y J. Tschudi, 1851).

Las piedras solían traerse de las canteras Muyna, Huacoto y Rumicolca, a 20 kilómetros del Cusco y para su transporte solo se usó la fuerza de los hombres, atándolas con cuerdas largas de cuero y rollizos de madera.

Se dice que para su construcción se mandó a traer unos veinte mil hombres de diferentes lugares del imperio; estos trabajaban por un tiempo limitado pues, posteriormente, se traían otros para reemplazarlos, de este modo, el trabajo resultaba más eficiente.

Estos trabajadores se organizaban en cuadrillas de la siguiente manera: mientras cuatro mil hombres estaban en las canteras extrayendo y trabajando las piedras, seis mil se encargaban de su transporte con el uso de maromas de cueros y de cabuyas. Los hombres restantes abrían zanjas para los cimientos y cortaban madera para las vigas que servirían de apoyo (Cieza de León, 1553). 


\section{Aspectos geotécnicos}

El área de estudio está conformada por rocas sedimentarias marinas, representada por la caliza y rocas de origen ígneo intrusivo, representada por la diorita; emplazadas en la llamada Meseta de Sacsayhuamán sobre la cual se fundaron, entre otros, la zona de 'Los Baluartes' (UNI, 2017).

El PAS se encuentra en una meseta - que se caracteriza por presentar un relieve plano con una serie de pequeñas colinas- con pendientes ligeros a fuertemente inclinadas (2-15\%). Está limitada por las elevaciones de Ccorao hacia el noreste, y hacia el suroeste limita con la depresión del Cusco. Estas unidades geomorfológicas están separadas por la Falla de Tambomachay (UNI, 2017).

Debido a la acción de esta falla, la roca se encuentra de muy fracturada a extremadamente fracturada, lo que produce inestabilidad en la zona donde se observa una serie de microdeslizamientos y deslizamientos de tamaño medio (UNI, 2017).

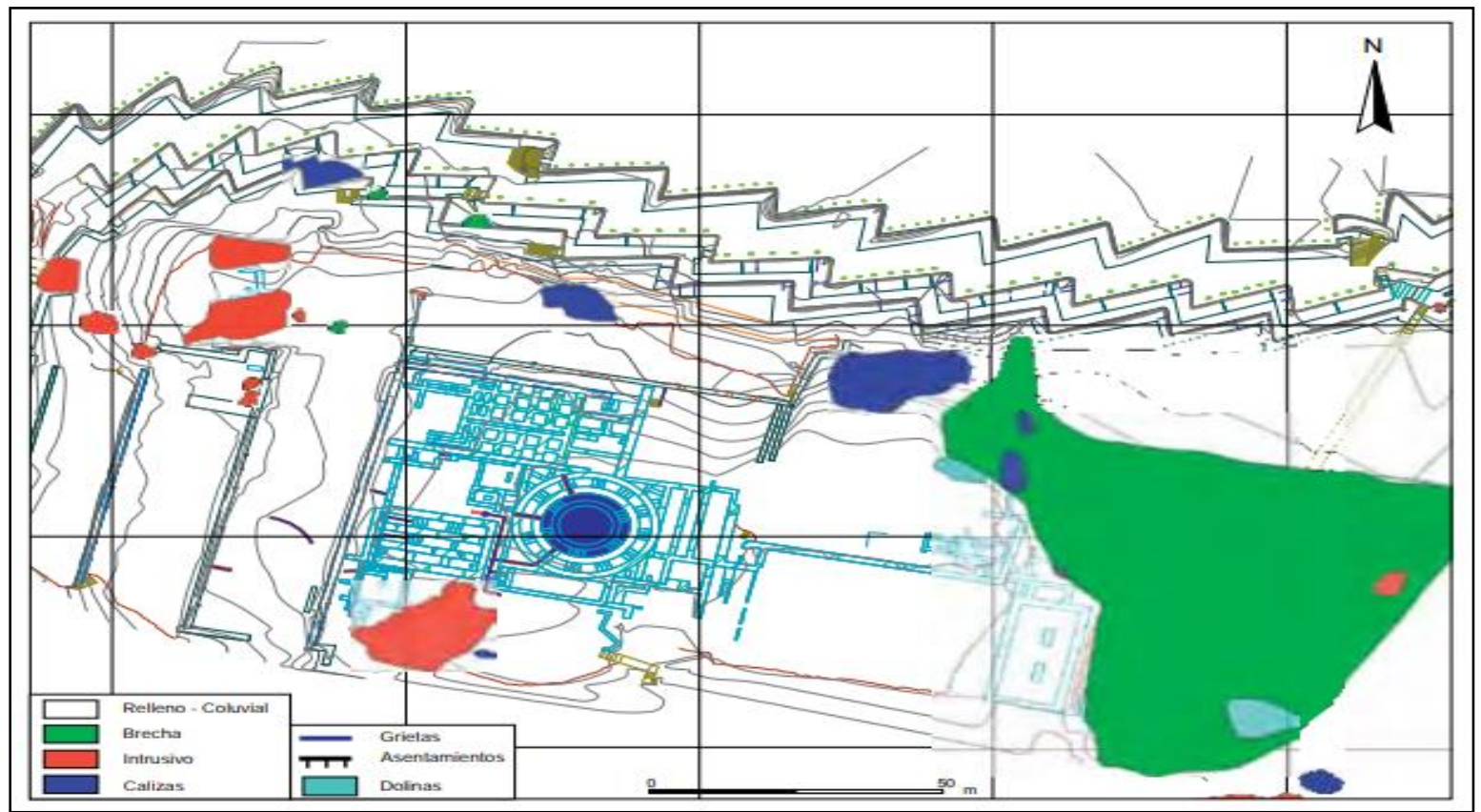

Figura 1. Mapa geológico y geodinámico de zona de Los Baluartes (Cárdenas, et al. 2009)

\section{Aspectos sísmicos}

Dentro del Reglamento Nacional de Edificaciones, en la norma E030 "Diseño Sismorresistente (2018)", indica que la zona de estudio se encuentra en la zona 2 con valor de Z, máximo de $0.25 \mathrm{~g}$; donde $\mathbf{g}$ es la aceleración de la gravedad.

\section{- Fallas activas}

La ciudad de Cusco está rodeada por fallas activas, la más cercana a la zona de estudio es la falla Tambomachay, se localiza a unos $4 \mathrm{~km}$ al norte de la ciudad.

\section{- Historia sísmica}

A continuación, se detallan los principales sismos que afectaron a la ciudad del Cusco: 
Tabla 6

Principales sismos en la ciudad de Cusco

\begin{tabular}{|l|c|c|}
\hline \multicolumn{1}{|c|}{ Fecha } & Magnitud (Ritcher) & Intensidad (MM) \\
\hline Abril 1986 & 5.2 & - \\
\hline Junio 1980 & - & IV \\
\hline Mayo 1965 & - & V - VI \\
\hline Noviembre 1961 & - & VI \\
\hline Marzo 1954 & - & IV \\
\hline Agosto 1952 & - & IV \\
\hline Febrero 1952 & - & V \\
\hline Mayo 1950 & - & VII \\
\hline Setiembre 1941 & - & VI - VII \\
\hline
\end{tabular}

Fuente: Bueno J. (2012)

\section{Metodología}

El método de la investigación es hipotético-deductivo, debido a que se inició con la observación del fenómeno a estudiar, luego se elaboraron hipótesis para explicar las variables planteadas y se dedujeron conclusiones, finalmente, se verificó la verdad de los enunciados. Se hace uso de una matriz de consistencia.

Además, este estudio tiene un diseño del tipo no experimental-transversal, debido a que no se construyó o simuló ninguna situación, sino que se observa el escenario existente.

\section{Análisis seudoestático del talud}

\section{Criterios de evaluación}

Con el objetivo de analizar la estabilidad del talud en la zona 'Los Baluartes', se siguieron los criterios de evaluación siguientes:

- Se contó con una correcta caracterización de la topografía de toda la zona.

- Fue necesario tener la ubicación específica de cada ensayo geotécnico y geofísico realizado anteriormente (2017) por la Universidad Nacional de Ingeniería.

- Se eligieron cinco secciones como muestra de la zona 'Los Baluartes'. Se priorizó su evaluación debido a que, por inspección visual, podría presentar peligro de inestabilidad.

- Los cortes elegidos debían estar a menos de 20 metros de los sectores donde anteriormente la Universidad Nacional de Ingeniería realizó ensayos geotécnicos (granulometría, densidad, DPL, etc.), y ensayos geofísicos (refracción sísmica, MASW, MAM).

- Se realizó el análisis de los resultados de los ensayos geotécnicos y geofísicos para la posterior caracterización geotécnica de las 5 secciones. 


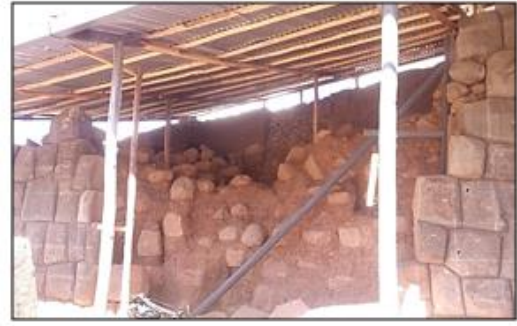

(a)

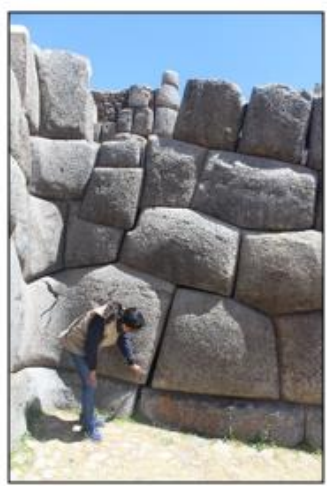

(d)

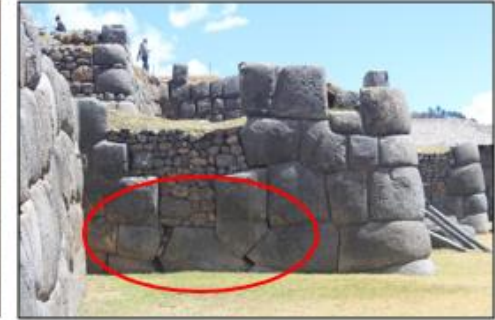

(b)

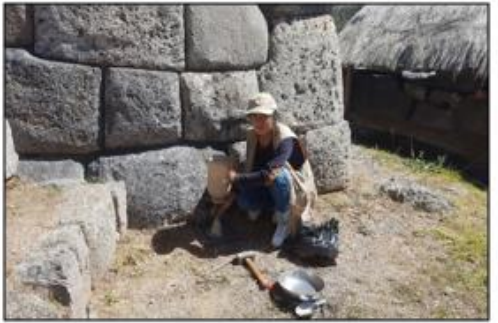

(c)

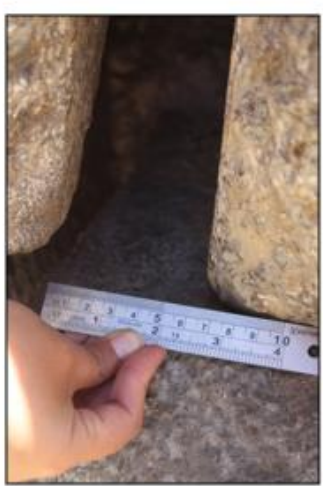

(e)

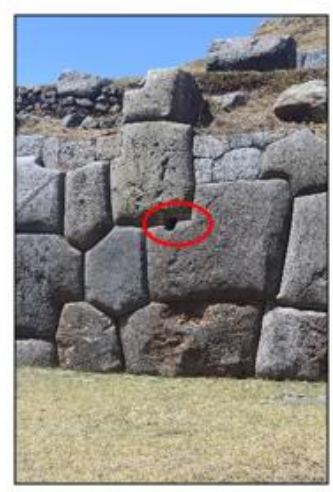

(f)

Figura 2: Análisis en campo: (a) muro colapsado ubicado en la tercera terraza de la zona Los Baluartes; (b) presencia de asentamiento y desplazamiento de muro; (c) realización de ensayo geotécnico; (d) inspección visual que evidencia empuje del talud; (e) medición del desplazamiento entre rocas del muro; (f) inspección de los sistemas de drenaje presentes.

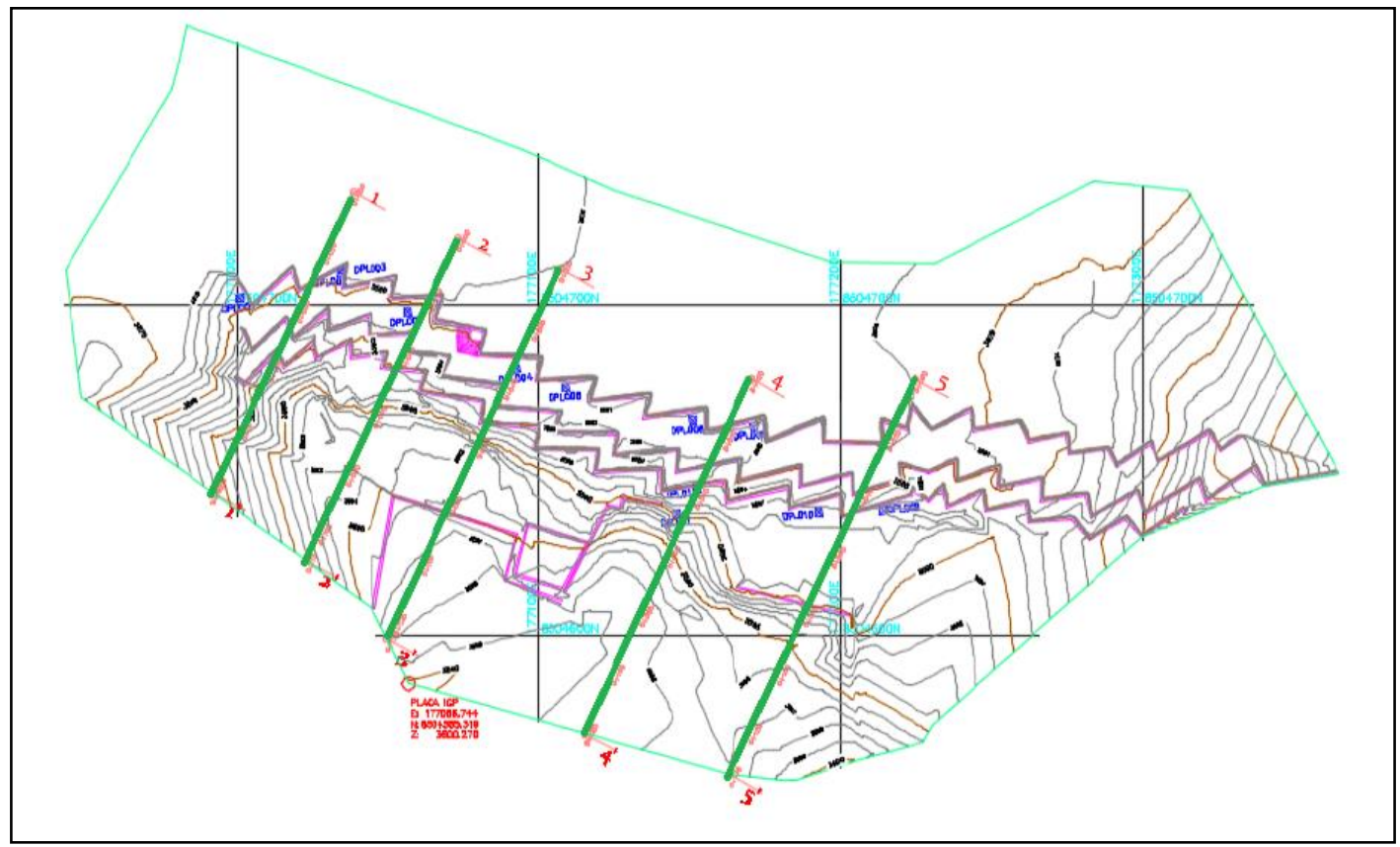

Figura 3: Secciones evaluadas, según criterio y conveniencia. Elaborado con Autocad Civil 3D 


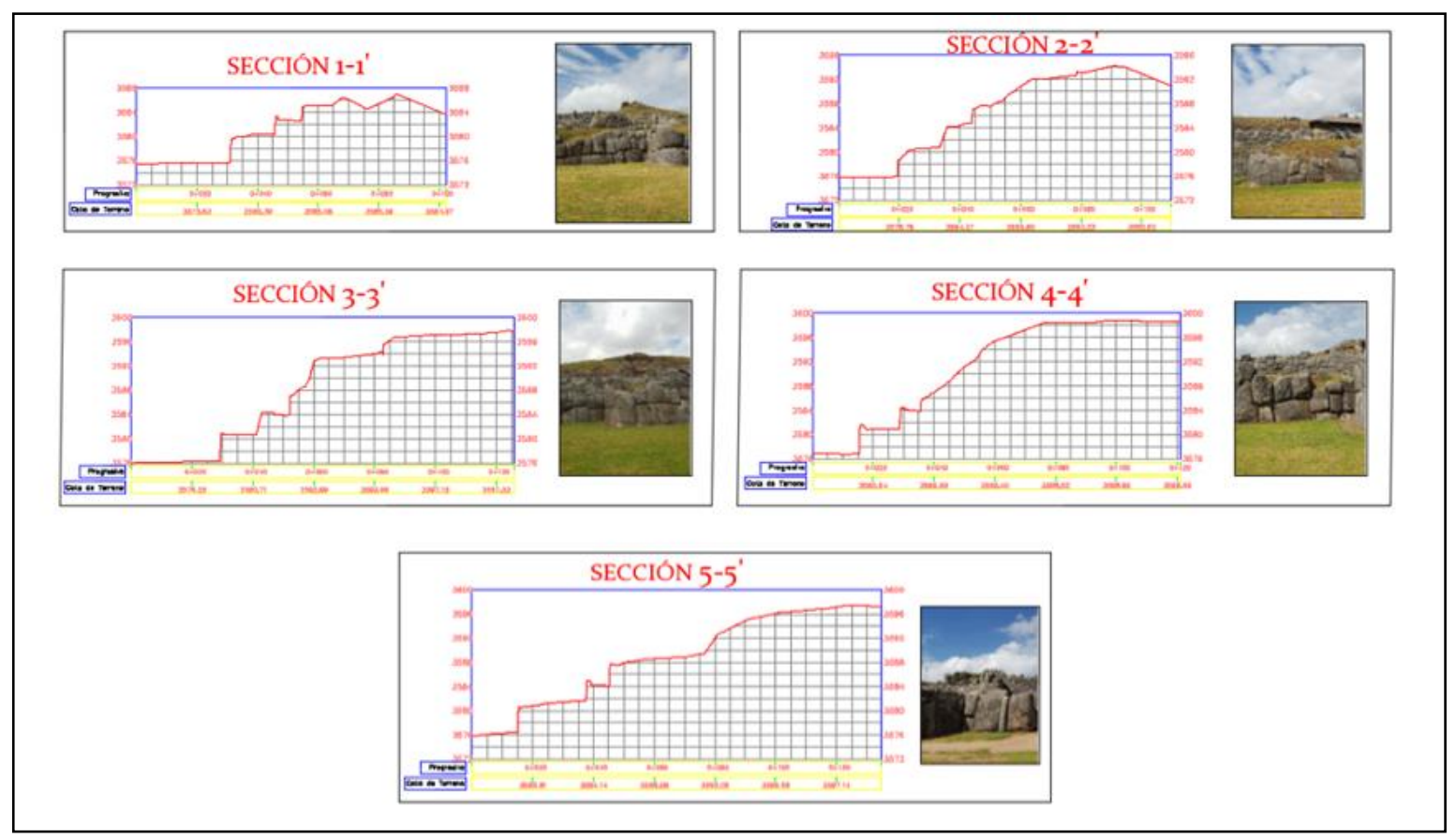

Figura 4: Perfiles correspondientes a las secciones elegidas, elaborado con Autocad Civil 3D

\section{Perfil sísmico y caracterización de los estratos}

Sobre la base de los ensayos geotécnicos y el mapa geológico del sector 'Los Baluartes', proporcionado por la Universidad Nacional de Ingeniería, se pudieron determinar los estratos presentes en el talud y, a partir de estos, se determinaron los parámetros de resistencia del suelo — tales como el ángulo de fricción y el peso unitario-. Asimismo, se hizo la tipificación del suelo en el perfil sísmico, según las velocidades de ondas de corte obtenidas del ensayo de refracción sísmica.

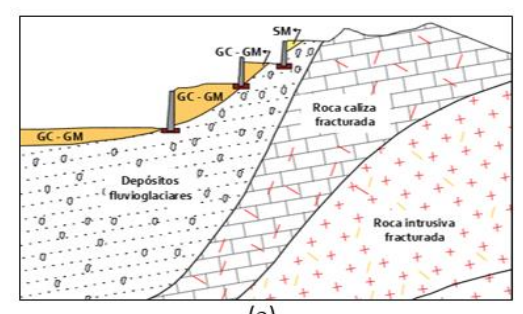

(a)

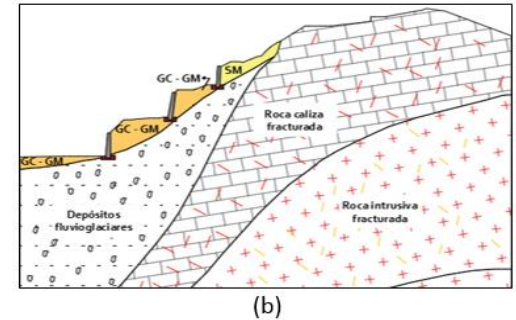

(b)

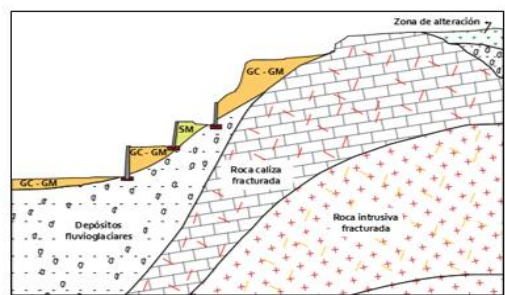

(c)

Figura 5: Perfiles estratigráficos de las secciones: (a) Sección 1; (b) Sección 2; (C)

Sección 3 
Tabla 2: Clasificación de suelos, sector Los Baluartes

\begin{tabular}{|c|c|c|}
\hline Sección & Terraza & Denominación \\
\hline \multicolumn{3}{|c|}{ Primer estrato } \\
\hline \multirow{3}{*}{$1-1$} & 1er Nivel & GC - GM, Grava Arcillosa limosa con arena \\
\hline & 2do Nivel & GC - GM, Grava Arcillosa limosa con arena \\
\hline & 3er Nivel & SM - SC, Arena Arcillosa limosa con grava \\
\hline \multirow{3}{*}{$2-2$} & 1er Nivel & GC - GM, Grava Arcillosa limosa con arena \\
\hline & 2do Nivel & GC - GM, Grava Arcillosa limosa con arena \\
\hline & 3er Nivel & SM - SC, Arena Arcillosa limosa con grava \\
\hline \multirow{3}{*}{$3-3$} & 1er Nivel & GC - GM, Grava Arcillosa limosa con arena \\
\hline & 2do Nivel & SM - SC, Arena Arcillosa limosa con grava \\
\hline & 3er Nivel & SM - SC, Arena Arcillosa limosa con grava \\
\hline \multirow{3}{*}{$4-4$} & 1er Nivel & GC - GM, Grava Arcillosa limosa con arena \\
\hline & 2do Nivel & SM - SC, Arena Arcillosa limosa con grava \\
\hline & 3er Nivel & GC - GM, Grava Arcillosa limosa con arena \\
\hline \multirow{3}{*}{$5-5$} & 1er Nivel & GC - GM, Grava Arcillosa limosa con arena \\
\hline & 2do Nivel & GC - GM, Grava Arcillosa limosa con arena \\
\hline & 3er Nivel & SM - SC, Arena Arcillosa limosa con grava \\
\hline \multirow{3}{*}{$6-6$} & 1er Nivel & GC - GM, Grava Arcillosa limosa con arena \\
\hline & 2do Nivel & SM - SC, Arena Arcillosa limosa con grava \\
\hline & 3er Nivel & GC - GM, Grava Arcillosa limosa con arena \\
\hline \multicolumn{2}{|c|}{ Suelo explanada } & GC - GM, Grava Arcillosa limosa con arena \\
\hline \multicolumn{2}{|c|}{ Suelo tratado } & GC, Grava Arcillosa \\
\hline \multicolumn{2}{|c|}{ Segundo estrato: depósito } & GC, Grava Arcillosa \\
\hline \multicolumn{2}{|c|}{ Tercer estrato } & Roca caliza fracturada \\
\hline \multicolumn{2}{|c|}{ Cuarto estrato } & Roca itrusiva fracturada \\
\hline
\end{tabular}

Tabla 3: Clasificación del sitio (Fuente: IBC (2012))

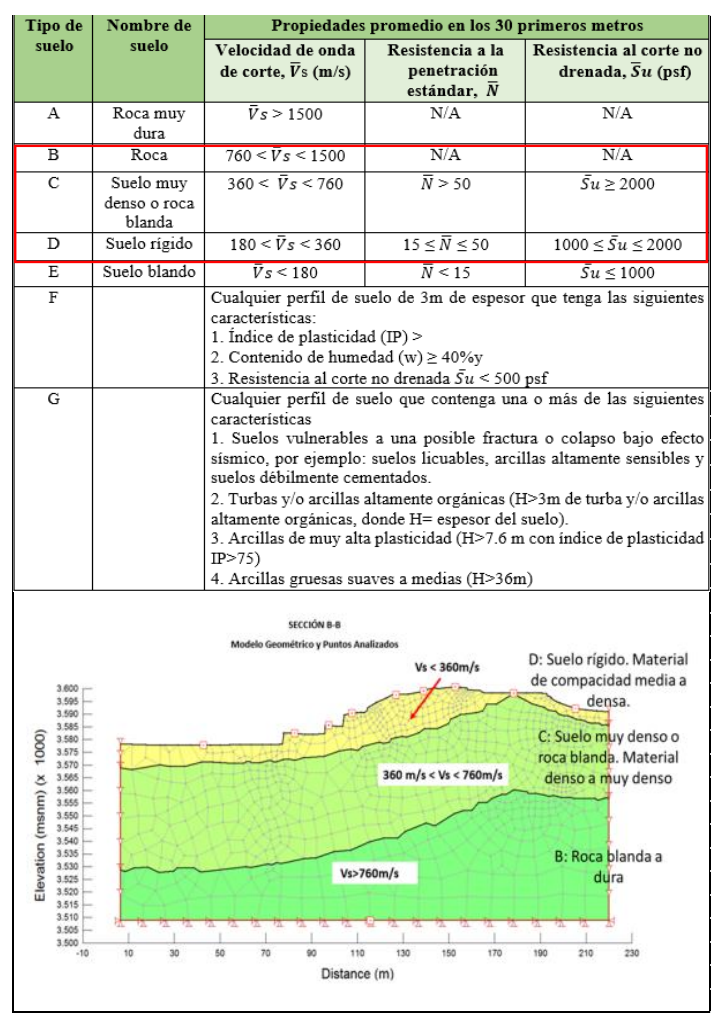


Además, se hizo uso de los resultados de los informes elaborados por la Dirección Desconcentrada de Cultura de Cusco (citado en la bibliografía), con el fin de corroborar los datos obtenidos del ensayo y completar los faltantes.

Para la determinación del parámetro de cohesión para el primer y segundo estrado, así como para el suelo tratado, que se encuentra en los cimientos de los muros, se utilizaron tablas de valores típicos de propiedades de los suelos. Además, se aplicó la ecuación de Kulhawy y Maine (1990), quienes proponen que se puede determinar la cohesión no drenada en función de los resultados del SPT ejecutados con una energía del 60\%, y se expresa en las unidades de la presión atmosférica $(\mathrm{Pa}=100 \mathrm{kN} / \mathrm{m} 2)$.

$$
\mathrm{Cu}=0,145(\mathrm{~N} 60)^{0.72}(\mathrm{~Pa})
$$

En cuanto a los estratos más profundos, al estar conformado por material rocoso; no presentan cohesión. Para la determinación de sus demás parámetros de resistencia al corte se hizo uso de la tabla 'Propiedades típicas del suelo y roca', propuesto por Casagrande, A:

Tabla 4: Propiedades típicas del suelo y roca

\begin{tabular}{|c|c|c|c|}
\hline Type and material & $\begin{array}{l}\text { Unit weight } \\
\text { (Saturated/dry) } \\
\mathrm{kN} / \mathrm{m}^{3}\end{array}$ & $\begin{array}{l}\text { Friction } \\
\text { angle (1) } \\
\text { degrees }\end{array}$ & $\begin{array}{l}\text { Cohesion } \\
\mathrm{kPa}\end{array}$ \\
\hline \multicolumn{4}{|l|}{ COHESIONLESS } \\
\hline \multicolumn{4}{|l|}{ Sand } \\
\hline Loose sand, uniform grain size & $19 / 14$ & $\begin{array}{l}28-34 \\
32-40\end{array}$ & \\
\hline $\begin{array}{l}\text { Dense sand, uniform grain size } \\
\text { Loose sand, mixed grain size }\end{array}$ & $\begin{array}{l}21 / 17 \\
20 / 16\end{array}$ & $=\begin{array}{r}32-40 \\
34-40\end{array}$ & \\
\hline \multirow{2}{*}{\multicolumn{4}{|c|}{$\begin{array}{l}\text { Dense sand, mixed grain size } \\
\text { Gravel }\end{array}$}} \\
\hline & & & \\
\hline Gravel, uniform grain size & $22 / 20$ & $34-37$ & \\
\hline \multirow{2}{*}{\multicolumn{4}{|c|}{$\begin{array}{l}\text { Compacted broken rock } \\
\text { Basals }\end{array}$}} \\
\hline & & & \\
\hline $\begin{array}{l}\text { Basalt } \\
\text { Chalk }\end{array}$ & $\begin{array}{l}22 / 17 \\
13 / 10\end{array}$ & $\begin{array}{l}40-30 \\
30-40\end{array}$ & \\
\hline Granite & $20 / 17$ & $45-50$ & \\
\hline Limestone & $19 / 16$ & $35-40$ & \\
\hline Sandstone & $17 / 13$ & $35-45$ & \\
\hline Shale & $20 / 16$ & $30-35$ & \\
\hline \multicolumn{4}{|l|}{ COHESIVE } \\
\hline \multicolumn{4}{|l|}{ Clay } \\
\hline Soft bentonite & $\begin{array}{l}13 / 6 \\
14 / 6\end{array}$ & $\begin{array}{r}7-13 \\
72-16\end{array}$ & $\begin{array}{l}10-20 \\
10-30\end{array}$ \\
\hline $\begin{array}{l}\text { Very soft organic clay } \\
\text { Soft slightly organic clay }\end{array}$ & $\begin{array}{l}14 / 6 \\
16 / 10\end{array}$ & $\begin{array}{l}12-16 \\
22-27\end{array}$ & $\begin{array}{l}10-30 \\
20-50\end{array}$ \\
\hline $\begin{array}{l}\text { Soft, slightly organic clay } \\
\text { Soft glacial clay }\end{array}$ & $17 / 12$ & $27-32$ & $30-70$ \\
\hline Stiff glacial clay & $20 / 17$ & $30-32$ & $70-150$ \\
\hline Glacial till, mixed grain size & $23 / 20$ & $32-35$ & $150-250$ \\
\hline \multicolumn{4}{|l|}{ Rock } \\
\hline $\begin{array}{l}\text { Hard igneous rocks: } \\
\text { granite, basalt, porphyry }\end{array}$ & ${ }_{25}^{(2)}$ to 30 & $35-45$ & $35000-55000$ \\
\hline $\begin{array}{l}\text { Metamorphic rocks: } \\
\text { quartzite, gneiss, slate } \\
\text { quadsedimentars rocks. }\end{array}$ & 25 to 28 & $30-40$ & $20000-40000$ \\
\hline limestone, dolomite, sandstone & 23 to 28 & $35-45$ & To00-30000 \\
\hline $\begin{array}{l}\text { oft sedimentary rock: } \\
\text { sandstone, coal, chalk, shale }\end{array}$ & 17 to 23 & $25-35$ & $1000-20000$ \\
\hline
\end{tabular}

Fuente: Casagrande, A. (1948)

Finalmente, se determinaron todos los parámetros de la resistencia al corte del suelo para todas las secciones, resultando lo siguiente: 
Tabla 5

Parámetros del suelo de resistencia al corte

\begin{tabular}{|c|c|c|c|c|c|c|}
\hline Sección & Terraza & $\begin{array}{l}\begin{array}{c}\text { Clasificación } \\
\text { (SUCS) }\end{array} \\
\end{array}$ & $\Phi$ & $\rho(g / c \mathrm{~cm} 3)$ & $(\mathbf{K N} / \mathbf{m} 3)$ & $\begin{array}{c}\mathrm{Cu} \\
(\mathrm{kN} / \mathrm{m} 2)\end{array}$ \\
\hline \multicolumn{7}{|c|}{ Primer estrato / material detrás de los muros incas } \\
\hline \multirow{3}{*}{$1-1$} & ler Nivel & GC-GM & 30.5 & 1.86 & 18.25 & 39.34 \\
\hline & 2do Nivel & GC-GM & 31.0 & 1.60 & 15.70 & 39.34 \\
\hline & $\begin{array}{l}3 \text { er Nivel } \\
\text { ler Nivel }\end{array}$ & $\frac{\text { SM-SC }}{\text { GC-GM }}$ & $\frac{32.0}{360}$ & $\frac{1.60}{1.86}$ & $\frac{15.70}{18.25}$ & $\frac{42.33}{73.15}$ \\
\hline \multirow[t]{2}{*}{$2-2$} & $\frac{\text { ler Nivel }}{\text { 2do Nivel }}$ & $\frac{G C-G M}{G C-G M}$ & $\frac{36.0}{31.0}$ & $\frac{1.86}{1.41}$ & $\frac{18.25}{13.83}$ & $\frac{73.15}{73.15}$ \\
\hline & 3er Nivel & SM-SC & 32.0 & 1.60 & 15.70 & 42.33 \\
\hline \multirow{3}{*}{$3-3$} & 1er Nivel & GC-GM & 38.2 & 1.86 & 18.25 & $\begin{array}{ll}72.55 \\
76.10\end{array}$ \\
\hline & 2do Nivel & SM-SC & 31.0 & 1.60 & 15.70 & 42.33 \\
\hline & 3er Nivel & SM-SC & 32.0 & 1.60 & 15.70 & 42.33 \\
\hline \multirow{3}{*}{$4-4$} & 1er Nivel & GC-GM & 34.5 & 1.86 & 18.25 & 58.86 \\
\hline & 2do Nivel & SM-SC & 31.0 & 1.60 & 15.70 & 42.33 \\
\hline & 3er Nivel & GC-GM & 36.9 & 1.16 & 11.38 & 69.06 \\
\hline \multirow{3}{*}{$5-5$} & $\frac{1 \text { er Nivel }}{2 \text { do Nivel }}$ & $\frac{G C-G M}{G C-G M}$ & 32.0 & 1.86 & $\frac{18.25}{15.70}$ & $\frac{58.86}{58.96}$ \\
\hline & 2 do Nivel & GC-GM & 31.0 & 1.60 & 15.70 & 58.86 \\
\hline & 3er Nivel & SM-SC & 31.6 & 1.60 & 15.70 & 42.33 \\
\hline $\begin{array}{l}\text { Todas las } \\
\text { secciones }\end{array}$ & $\begin{array}{c}\text { Suelo } \\
\text { explanada }\end{array}$ & GC-GM & 31.0 & 1.50 & 14.72 & 58.86 \\
\hline \multicolumn{7}{|c|}{ Seggundo estrato } \\
\hline $\begin{array}{l}\text { Todas las } \\
\text { secciones }\end{array}$ & $\begin{array}{l}\text { Segundo } \\
\text { estrato }\end{array}$ & GC & 36.9 & 1.16 & 11.38 & 69.06 \\
\hline \multicolumn{7}{|c|}{ Suelo tratado } \\
\hline $\begin{array}{l}\text { Todas las } \\
\text { secciones }\end{array}$ & Suelo tratado & GC & 28.0 & 1.58 & 15.50 & 76.10 \\
\hline \multicolumn{7}{|l|}{ secciones } \\
\hline Sección & Terraza & Clasificación & $\Phi$ & $\rho(g / c m 3)$ & $(\mathrm{KN} / \mathrm{m} 3)$ & $\mathrm{Cu}(\mathrm{kN} / \mathrm{m} 2)$ \\
\hline \multicolumn{7}{|c|}{ Tercer estrato } \\
\hline $\begin{array}{l}\text { Todas las } \\
\text { secciones }\end{array}$ & $\begin{array}{c}\text { Segundo } \\
\text { estrato }\end{array}$ & Roca caliza & 39.9 & - & 25.5 & 0 \\
\hline \multicolumn{7}{|c|}{ Cuarto estratc } \\
\hline $\begin{array}{l}\text { Todas las } \\
\text { secciones }\end{array}$ & $\begin{array}{c}\text { Segundo } \\
\text { estrato }\end{array}$ & $\begin{array}{c}\text { Roca intrusiva: } \\
\text { diorita }\end{array}$ & 45.0 & - & 27.66 & 0 \\
\hline
\end{tabular}

\section{Determinación de la conductividad hidráulica y de la infiltración}

\section{Conductividad hidráulica}

Para determinar la estabilidad del talud considerando la incidencia de la infiltración causada por la precipitación, se necesita establecer parámetros, como la conductividad hidráulica. Este se obtiene mediante tablas de valores típicos de la conductividad hidráulica, donde se relaciona el tipo de suelo con su valor de conductividad.

Dichas tablas son de autores como Coduto (1999), Doménico y Schwartz (1990) y Sotelo G. (2002); por tanto, obtenemos que:

- Para el suelo (GC, GM) el valor de conductividad hidráulica es de $4 \mathrm{e}-7 \mathrm{~m} / \mathrm{s}$.

- Para el suelo (SM) el valor de conductividad hidráulica es de $1 \mathrm{e}-4 \mathrm{~m} / \mathrm{s}$.

- Para el material rocoso el valor de conductividad hidráulica es de 1 e-9 m/s.

\section{Infiltración}

El programa Slide, donde se procesan los datos, indica que las unidades de infiltración son $\mathrm{m} / \mathrm{seg}$. Esto se debe a que la infiltración representa un volumen de fluido entrando (o saliendo) del talud.

Al no tener datos de la escorrentía en el lugar, se asume que la infiltración viene a ser igual a la precipitación máxima dada por 24 horas en $\mathrm{m} / \mathrm{s}$.

De la recolección de los registros de la estación pluviométrica de Perayoc, se determina que el valor de infiltración es 54,40 mm/día, equivalente a 6,2963 e-7 m/s., el cual corresponde a la precipitación máxima en 24 horas para el mes de febrero del año 2013. 


\section{Procesamiento en el programa Slide V6.0}

Para el análisis de estabilidad de taludes, se hizo uso del programa Slide V6.0; primero, sin considerar la carga sísmica y sin la presencia infiltración; posteriormente, se consideró esta condición.

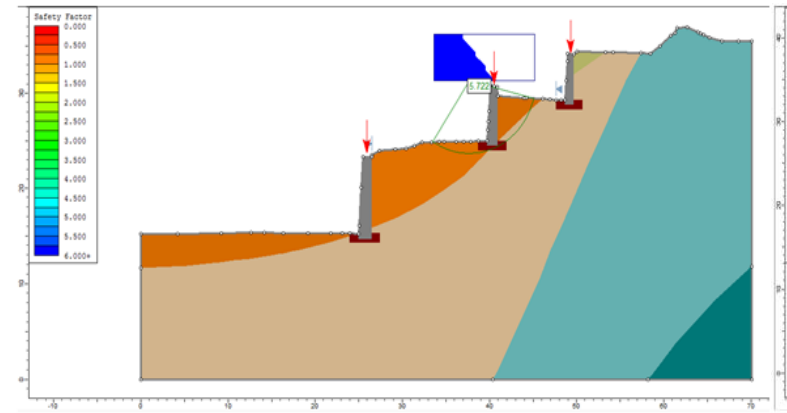

(a)

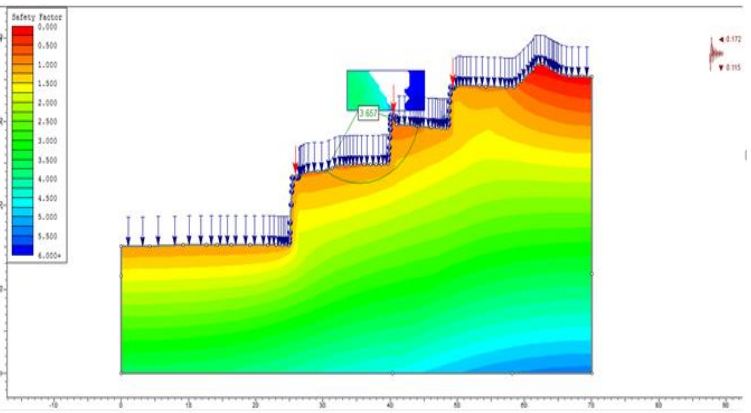

(b)

Figura 6: Análisis en el programa Slide: (a) sin considerar la infiltración; (b) considerando infiltración y condición seudoestática.

\section{Resultados}

Después de determinar los parámetros necesarios, ejecutamos el análisis de estabilidad en condiciones seudoestáticas y se introdujo el valor de la infiltración. Los estudios fueron realizados por el método de equilibrio límite y, para este procedimiento, se utilizó el software Slide V 6.0. El método elegido para el cálculo de factores de seguridad fue el de Bishop simplificado.

Tabla 6

Resultado del factor de seguridad para todas las secciones en diferentes condiciones

\begin{tabular}{|c|c|c|c|c|c|c|c|c|c|c|c|c|c|c|c|}
\hline \multirow[b]{2}{*}{ Condición } & \multicolumn{5}{|c|}{ Primera Terraza } & \multicolumn{5}{|c|}{ Segunda Terraza } & \multicolumn{5}{|c|}{ Tercera Terraza } \\
\hline & $\begin{array}{c}\text { Secc. } \\
1-1\end{array}$ & $\begin{array}{c}\text { Secc. } \\
2-2\end{array}$ & $\begin{array}{c}\text { Secc. } \\
3-3\end{array}$ & $\begin{array}{c}\text { Secc. } \\
4-4\end{array}$ & $\begin{array}{l}\text { Secc. } \\
5-5\end{array}$ & $\begin{array}{c}\text { Secc. } \\
1-1\end{array}$ & $\begin{array}{c}\text { Secc. } \\
2-2\end{array}$ & $\begin{array}{c}\text { Secc. } \\
3-3\end{array}$ & $\begin{array}{c}\text { Secc. } \\
4-4\end{array}$ & $\begin{array}{c}\text { Secc. } \\
5-5\end{array}$ & $\begin{array}{c}\text { Secc. } \\
1-1\end{array}$ & $\begin{array}{c}\text { Secc. } \\
2-2\end{array}$ & $\begin{array}{c}\text { Secc. } \\
3-3\end{array}$ & $\begin{array}{c}\text { Secc. } \\
4-4\end{array}$ & $\begin{array}{l}\text { Secc. } \\
5-5\end{array}$ \\
\hline $\begin{array}{l}\text { Condición } \\
\text { estática }\end{array}$ & 3.50 & 3.84 & 4.00 & 3.73 & 3.22 & 5.72 & 5.39 & 4.96 & 4.75 & 5.39 & 7.37 & 4.49 & 2.13 & 2.87 & 5.05 \\
\hline $\begin{array}{c}\text { Condición } \\
\text { psendoestática }\end{array}$ & 2.60 & 2.81 & 2.89 & 2.69 & 2.32 & 4.06 & 3.84 & 3.88 & 3.65 & 4.05 & 5.21 & 3.36 & 1.62 & 1.43 & 3.55 \\
\hline $\begin{array}{c}\text { Condición } \\
\text { pseudoestática, y } \\
\text { con infiltración }\end{array}$ & 2.11 & 2.35 & 2.37 & 2.18 & 1.84 & 3.66 & 3.40 & 3.55 & 3.00 & 3.58 & 4.40 & 2.89 & 0.99 & 0.93 & 3.42 \\
\hline
\end{tabular}

De todas las secciones evaluadas se observa que existe inestabilidad en la tercera terraza de la sección 3 y 4 . Se determinó un factor de seguridad (FS) de 0,99 y 0,93 respectivamente. Esto concuerda con la inspección visual que se realizó en campo, donde se determinaron los muros con mayor presencia de desplazamiento y daño por empuje.

\section{Conclusiones}

- El factor de seguridad calculado en el escenario más crítico para la tercera terraza en las secciones 01, 02 y 05; es mayor a 1,25 (FS $=4,40,2,89$ y 3,42 , respectivamente), por tanto, la tercera terraza en las secciones mencionadas es estable. Mientras que el factor de seguridad de la tercera terraza calculado para las secciones 03 y 04 del talud son menores a 1,25 (FS = 0,99 y 0,93; respectivamente). Según esta característica se indica que el talud de la tercera terraza en las secciones 03 y 04 es inestable. 
- En concordancia con investigaciones anteriores se llega a la conclusión: que el factor con mayor incidencia en la inestabilidad del talud y colapso de muros en el sector 'Los Baluartes' es la precipitación pluvial, puesto que, debido a la saturación del suelo, la resistencia al corte del mismo, disminuye.

- A lo largo del talud de la zona de 'Los Baluartes', actúan fuerzas expansivas de origen hidrogeológico, sísmico y de carga. Se adiciona la presencia de planos de deslizamientos del tipo rotatorio. Esta actividad en el talud, genera desplazamientos en varias direcciones y, por tanto, la separación de los líticos en los muros, lo que finalmente causará el colapso de los mismos.

- Se observa en toda la estructura de los muros del sector 'Los Baluartes', un proceso continuo de separación entre líticos; la que varía de pocos milímetros hasta diez centímetros. Esto constata el proceso de movimiento del talud.

- Se recomienda realizar un estudio de la estabilidad del talud -incluyendo el modelamiento de la geometría de los canales incas y no incas (transversales y longitudinales) - para corroborar la influencia de la presencia de los mismos en la estabilidad del talud y de los muros.

- Se recomienda que en los estudios de estabilidad de taludes no solo se incluyan parámetros de resistencia del suelo, sino que también se incluyan sus parámetros hídricos, puesto que se mostró su influencia en la estabilidad.

\section{Discusión}

La presente investigación ¿de qué manera aporta cognoscitivamente a la ingeniería civil?

El presente proyecto, si bien pertenece a la rama de la geotecnia — porque analiza la estabilidad del talud en la zona Los Baluartes del PAS-, también requiere de las bases de la hidrología y sismicidad para su entendimiento. Esta investigación 'Los Baluartes' e impulsa el estudio de monumentos históricos desde un enfoque ingenieril, ya que actualmente no existen muchos profesionales en ingeniería civil que se dediquen a su análisis y conservación.

\section{Agradecimientos}

Agradezco al decano de la Universidad Nacional de Ingeniería, Dr. Jorge Alva Hurtado, quien permitió el desarrollo de la investigación al compartir los datos y resultados del estudio elaborado anteriormente por dicha casa de estudios en la zona de Sacsayhuamán, en los años 2016 y 2017.

A mi querida tía y mentora, MSc. ing. Carmen Ortiz Salas, quien fue pieza fundamental en todo el proceso de la investigación, por ser la principal fuente de apoyo y conocimiento para el desarrollo de la misma.

\section{Referencias bibliográficas}

Alva J., Ortiz C., Pérez A., Soto J. \& Riveros G. (2016). Análisis dinámico bi-dimensional de amplificación sísmica en el Parque Arqueológico de Sacsayhuamán. World Engineering Conference on Disaster Risk Reduction. Lima, Perú.

Alva J., Ortiz C., Pérez A. \& Soto J. (2015). Mediciones geofísicas en el Parque Arqueológico de Sacsayhuamán. XIX Hernández R., Fernández C. \& Baptista P. (2010). Metodología de Congreso Nacional de Ingeniería Civil. Lima, Perú.

Bueno J. (2012). Actividad geodinámica en los baluartes del Jarriel T. (2016). Using 3D terrace modeling to preserve the incan Parque Arqueológico de Sacsayhuamán Cusco. Cusco, Perú.

Carlotto V., Cárdenas J. \& Carlier G. (2011). Geología del Lohr K. (2014). Restoring and preserving the incan cultural wonder cuadrángulo de Cusco 28-s - 1:50 000, Boletín N. ${ }^{\circ} 138$, Serie A. Lima: INGEMMET.

Dirección Regional de Cultura - Cusco (2007). Estudios Mar R. \& Beltrán Caballero J. (2014). El conjunto arqueológico de fundamentales: Sacsayhuamán. Lima: Dirección Regional de Cultura Cusco.
Dirección Regional de Cultura Cusco (2010). Informe geotécnico de Los Baluartes del Sitio Arqueológico de Sacsayhuamán. Cusco. Perú.

Dirección Regional de Cultura Cusco (2010). Informe final del expediente técnico de actividad $N^{\circ}{ }^{\circ}$ 03-PMPAS-PAS. Cusco. Perú.

la investigación. México D.F.: McGraw Hill Editorial. Virginia, EEUU

of Sacsayhuamán. (Tesis de maestría). Universidad de Virginia, EEUU.

Sacsayhuamán (Cusco): una aproximación a su arquitectura. España: Revista Española de Antropología Americana, vol. 44, núm. 1, 9-38. 
International Code Council (2012). International building code. Suárez, J. (2009). Deslizamientos. Análisis geotécnico. Colombia: EEUU: International Code Council ICC.

Servicio Nacional de Capacitación para la Industria de la Instituto de investigaciones sobre erosión y Construcción - SENCICO (2010). Reglamento Naciona de Edificaciones. Lima: Apolo Editorial. deslizamientos.

Universidad Nacional de Ingeniería (UNI). (2017). Informe de geología de la zona de Los Baluartes del Sitio Arqueológico de Sacsayhuaman. Lima: UNI. 